Check for updates

Cite this: Chem. Sci., 2020, 11, 195

๑ All publication charges for this article have been paid for by the Royal Society of Chemistry

Received 9th September 2019

Accepted 5th November 2019

DOI: $10.1039 /$ c9sc04555d

rsc.li/chemical-science

\section{Novel four-disulfide insulin analog with high aggregation stability and potency $\dagger$}

\author{
Xiaochun Xiong, (D) $\ddagger^{\mathrm{a}}$ Alan Blakely, (D) $\ddagger^{\mathrm{a}}$ Prasoona Karra, ${ }^{\mathrm{b}}$ Michael A. VandenBerg, ${ }^{\mathrm{c}}$ \\ Gabrielle Ghabash, ${ }^{a}$ Frank Whitby, ${ }^{a}$ Yi Wolf Zhang, ${ }^{a}$ Matthew J. Webber, (D) \\ William L. Holland, ${ }^{\text {b }}$ Christopher P. Hill (D) *a and Danny Hung-Chieh Chou (D) *a
}

\section{Introduction}

Insulin is a peptide hormone that regulates blood glucose metabolism and is an indispensable therapeutic for treating diabetes for millions of people worldwide. ${ }^{1}$ Human native insulin comprises two chains: A chain with 21 amino acid residues (A1-21) and B chain with 30 amino acid residues (B130). ${ }^{2}$ The two chains are connected by two inter-chain disulfide bonds (A7-B7 and A20-B19) and one intra-A-chain disulfide (A6A11). Human insulin forms hexamers in the presence of zinc ions through coordination of B10 His. In all commercially available insulin formulations, the hexamer, which is relatively stable, is the dominant form. However, even with the presence of three disulfides and the hexameric form, human insulin is still prone to formation of aggregated fibrils, which makes continuous refrigeration necessary to ensure quality. ${ }^{3}$ Thus, there is a need to further increase insulin stability to provide an analog that does not require continuous refrigeration.

All known vertebrate insulins have a conserved threedisulfide skeleton. ${ }^{4}$ There are some examples of insulin-like

\footnotetext{
${ }^{a}$ Department of Biochemistry, University of Utah, Salt Lake City UT 84112, USA. E-mail: chris@biochem.utah.edu;dchou@biochem.utah.edu

${ }^{b}$ Department of Nutrition and Integrative Physiology, University of Utah, Salt Lake City UT 84112, USA

'Department of Chemical \& Biomolecular Engineering, University of Notre Dame, IN 46556, USA

$\dagger$ Electronic supplementary information (ESI) available: Detailed synthesis procedure and mass spectrometry data along with biological experiment protocol. See DOI: 10.1039/c9sc04555d

$\ddagger$ These authors contribute equally.
}

peptides from invertebrates with four disulfides, although their aggregation stability was not well characterized..$^{5,6}$ Introduction of additional disulfides into a protein may lead to higher stability, ${ }^{7}$ and an insulin analog with an additional disulfide between A10 and B4 has been described. ${ }^{8}$ This A10-B4 linkage was chosen because the two $\mathrm{C}_{\alpha}$ were in close $(5.4 \AA)$ proximity, and crystal structure determination of the A10-B44SS-Ins hexamer revealed that the extra disulfide has only minor impacts on tertiary structure. Importantly, A10-B4-4SSIns has a similar binding affinity as native insulin but has a higher aggregation and thermal stability, which is a promising feature for insulin therapeutics. ${ }^{89}$ In an analogous study, the DiMarchi group recently synthesized another four-disulfide insulin-like peptide, Con-Ins G2, with the extra disulfide between the human A4 position and an N-terminal extended B chain (human $\mathrm{B}^{\prime \prime}-6^{\prime \prime}$ position) and showed that Con-Ins G2 is inactive. ${ }^{6}$ They further performed disulfide scanning to identify a number of other additional disulfide bonds that result in reduced bioactivity. ${ }^{10}$ These observations indicate that an additional disulfide may lead to higher insulin stability but that the extra disulfide may compromise bioactivity.

Guided by our studies of novel venom insulin molecules from fish-hunting cone snails,${ }^{11}$ we hypothesized that an additional disulfide may be inserted into native insulin between an extended A-chain and B-chain residues B21-B23. Here, we report the chemical synthesis and characterization of 4 insulin analogs with additional disulfide bonds in this region. We further evaluated the properties of A22-B22-4SS-Ins, and identified it as a novel four-disulfide insulin analog with high stability and activity. 


\section{Results and discussion}

\section{Design of 4SS-Ins molecules}

Efforts to incorporate an extra disulfide between C-terminally extended A chain residues and B21-B23 have not been reported. Indeed, the extended A-chain residues of the insulin relatives IGF-1 and IGF-2 point away from the B chain..12,13 However, our studies of cone snail venom insulin molecules suggested that this might be a viable approach. Specifically, venom insulins Con-Ins-K1 and K2 have a C-terminally extended A chain (24 residues) and truncated B chain (22 residues), and modeling studies suggested that the extended A chain may point toward the C-terminus of the B chain. ${ }^{\mathbf{1 1}} \mathrm{We}$ therefore designed insulin analogs that include a fourth disulfide bond in this region.

Using two independent insulin crystal structures (PDB 1EVR and $1 \mathrm{ZNJ}),{ }^{14}$ we found that the distance of the two $\mathrm{C}_{\alpha}$ for both A21-B22 and A21-B23 is $\sim 6 \AA$, which is borderline for the introduction of a disulfide bond (Fig. 1). Our designs therefore included analogs with an extra C-terminal residue, A22, to provide more flexibility for the additional disulfide. Based on the modeling, we decided to investigate the structure-activity relationship of four analogs that displayed the additional disulfides: A22-B21, A22-B22, A22-B23 and A21-B22.

\section{Synthesis of 4SS-Ins molecules}

Our synthetic route toward four-disulfide insulin molecules is described in Scheme 1. First, the protected A chain [A6, 7, 11, 22-Acm] A1 was synthesized on 2-chlorotrityl chloride (2-CTC) resin through solid phase peptide synthesis (SPPS). Note that the A8-A9 dipeptide was synthesized as an isoacylpeptide to improve A chain solubility (Bold residues in Scheme 1). ${ }^{15} \mathbf{A 1}$ was then treated with a cocktail of TFA/TIPS/ $\mathrm{H}_{2} \mathrm{O}$ to yield the corresponding four Acm-protected product A2 in 24\% yield. A chain $[\mathrm{A} 6,7,11,21-\mathrm{Acm}] \mathbf{A 3}$ was obtained in 26\% yield using the same route (Scheme 2). Next, protected B chain [B7, 21-Acm] B1 was generated through SPPS and activated with DTDP during acidic cleavage to give the [B7, 21-Acm, B19-SPy] chain B2 in $24 \%$ yield. B chains [B7, 22-Acm, B19-Spy] B3 and [B7, 23-Acm,

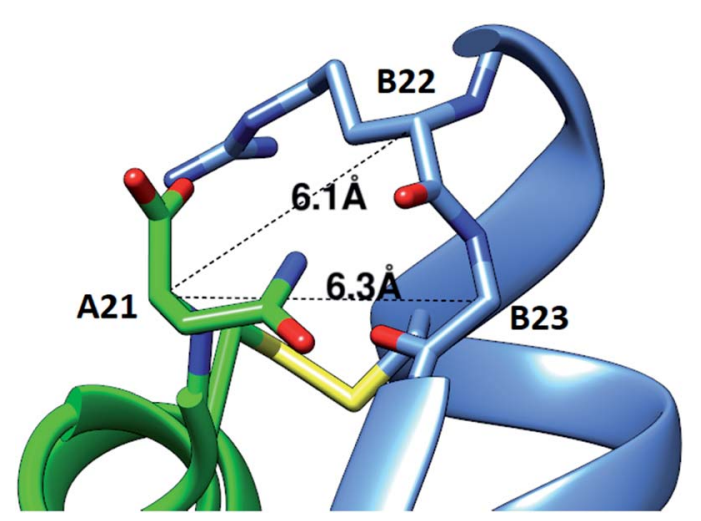

Fig. 1 Modeling of a fourth disulfide bond in insulin. The crystal structure of $\mathrm{R}_{6}$ insulin (PDB: 1EVR) is shown with $\mathrm{C}_{\alpha}$ distances between $\mathrm{A} 21$ and nearby residues on the $\mathrm{B}$-chain indicated.
B19-Spy] B4 were synthesized in 25\% and 21\% yield respectively (Scheme 2).

With both A and B chains in hand, we attempted to form the four disulfide bonds through a two-step process. First, A2 and B2 were dissolved and stirred in $6 \mathrm{M}$ urea containing $0.2 \mathrm{M}$ $\mathrm{NH}_{4} \mathrm{HCO}_{3}(\mathrm{pH}$ 7.5) buffer solution. The reaction reached complete conversion of $\mathbf{A 2}$ in 10 min and yielded a heterodimer C1 in 68\% yield based on the amount of A2 after HPLC purification. Conversion of C1 to A22-B21-4SS-Ins was smoothly achieved by treatment with excess amount of iodine in methanol, and the A6-A11, A7-B7 and A22-B21 disulfide bonds were formed through in situ Acm deprotection/disulfide formation. The product A22-B21-4SS-Ins was obtained in 11\% yield after purification (Scheme 1). Three additional analogs A22-B22-4SSIns, A22-B23-4SS-Ins and A21-B22-4SS-Ins were obtained in $4.9 \%, 9.1 \%$ and $6.4 \%$ yield, respectively, over two steps (Scheme 2).

\section{A22-B22-4SS-Ins displays native activity and resistance to aggregation}

A phospho-AKT (Ser 473) insulin signaling stimulation assay was used to evaluate insulin bioactivity of the four analogs (Fig. 2A). Native insulin and A22-B22-4SS-Ins displayed similar potency $\left(\mathrm{EC}_{50} 3.86 \mathrm{nM}\right.$ vs. $2.87 \mathrm{nM}$, respectively). In contrast, both A22-B21-4SS-Ins and A22-B23-4SS-Ins have much reduced potency (50- and 20-fold reduction respectively), and A21-B224SS-Ins is 4 -fold less potent than native insulin. These results indicate that the relatively close proximity between A21 and B22 can lead to partially preserved bioactivity, and the extra flexibility provided by the $\mathrm{A} 22$ residue results in close to native potency for the A22-B22-4SS-Ins analog. Consistent with the cell-based assay, an insulin tolerance test in mice showed that A22-B22-4SS-Ins and native insulin display similar in vivo activity following subcutaneous injection (Fig. 2B).

We further evaluated propensity for aggregation using a stressed aging assay of continuous agitation in $\mathrm{pH} 7.4$ buffer at $37^{\circ} \mathrm{C}$. As shown in Fig. 2C, native insulin behaves similarly to previous reports as it aggregated in less than 10 hours of shaking, ${ }^{16}$ while A22-B22-4SS-Ins did not reach an equivalent level of aggregation until at least 42 hours of shaking. This result demonstrates that A22-B22-4SS-Ins has a superior effect over native insulin in preventing aggregation, a key factor for insulin formulation.

\section{A22-B22-4SS-Ins crystal structure and comparison to native insulin}

To investigate the structural basis of the high stability and potency, crystals of A22-B22-4SS-Ins were grown by sitting-drop vapor diffusion. The crystals contained one insulin hexamer in the asymmetric unit. The hexamer is bound by seven phenol molecules occupying the six classical phenolic binding sites, and one additional site. Also present are two $\mathrm{Zn}^{2+}$ ions that, together with the phenol, stabilize the hexameric $\mathrm{R}_{6}$ conformation of the insulin (Fig. 3). Phenolic compounds have been well documented for their use in stabilizing insulin hexamers, ${ }^{17,18}$ and under our screen conditions the addition of phenol 


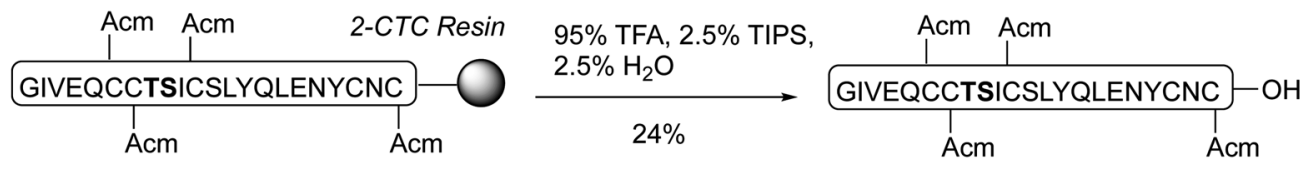

A1

A2

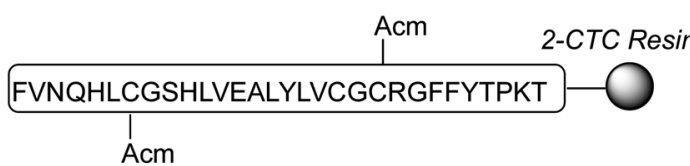

B1

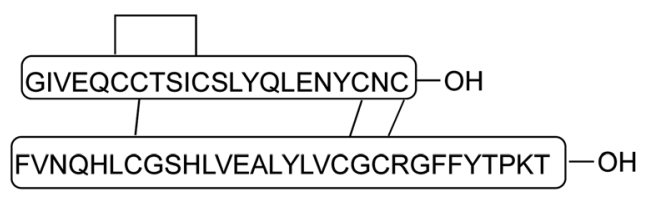

A22-B21-4SS-Ins
95\% TFA, 2.5\% TIPS, $2.5 \% \mathrm{H}_{2} \mathrm{O}, 15$ eq. DTDP $24 \%$

\section{$\rightarrow F V$}

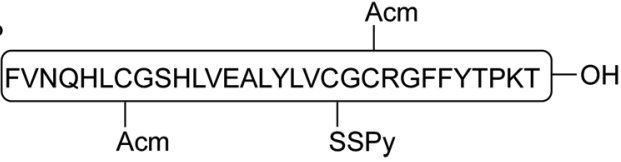

B2

0.8 eq. of A2, $0.2 \mathrm{M} \mathrm{NH}_{4} \mathrm{HCO}_{3}$, $6 \mathrm{M}$ Urea, $\mathrm{pH} 7.5$

$68 \%$ based on $\mathrm{A2}$
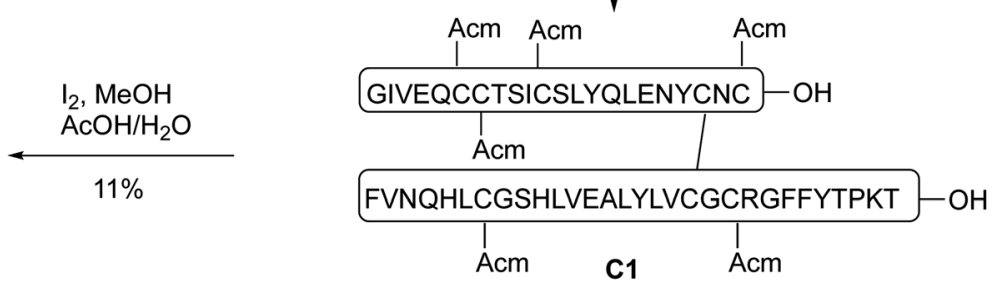

Scheme 1 Total chemical synthesis of A22-B21-4SS-Ins analog.

was found to be essential for the formation of crystals. Electron density for the fourth disulfide bond connecting the $\mathrm{C}$-terminus of the A chain to the type-I $\beta$-turn on the B-chain is well defined
(Fig. 4), and the overall structure reveals that the insulin backbone is able to accommodate the new disulfide linkage without appreciable conformational changes. The average root mean

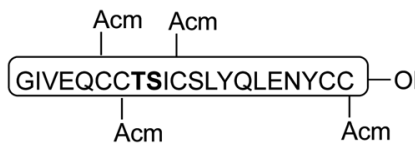

A3: $26 \%$

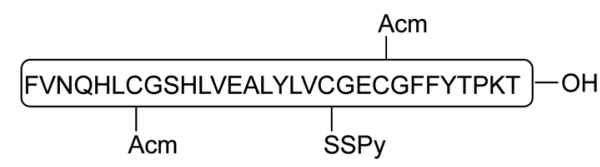

B3: $25 \%$

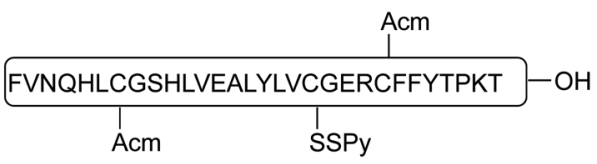

B4: $21 \%$

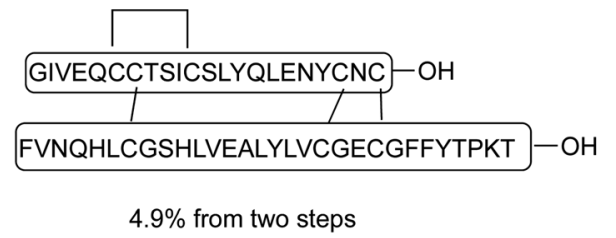

A22-B22-4SS-Ins

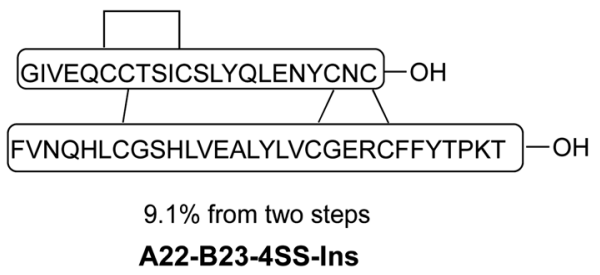

A22-B23-4SS-Ins

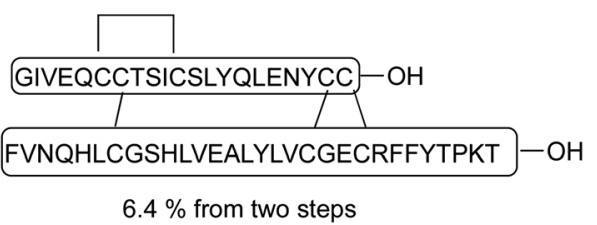

A21-B22-4SS-Ins

Scheme 2 Synthesis of three additional analogs using the same synthetic route described in scheme 1. 
A

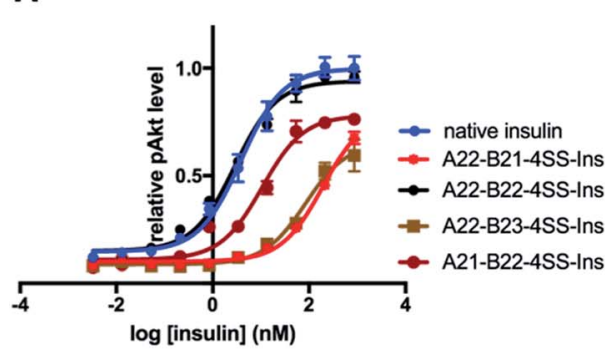

B

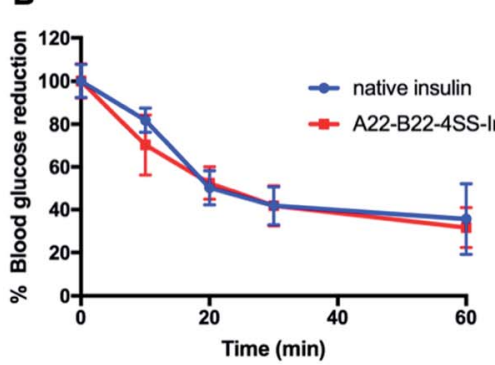

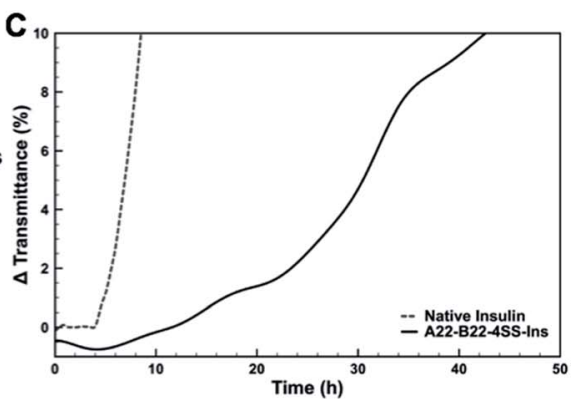

Fig. 2 Characterization of novel four-disulfide insulin analogs. (A) Insulin bioactivity profile compared to native insulin. Data represent the average of 4 independent measurements. Error bars represent the standard deviation. (B) Insulin tolerance test in mice. Data represent the average of 4 mice. Error bars represent the standard deviation. (C) Kinetic profiling of aggregation, monitoring change in transmittance (i.e., increase in turbidity) at $1 \mathrm{mg} \mathrm{ml}^{-1}$ under continuous agitation at $37^{\circ} \mathrm{C}$ in $\mathrm{pH} 7.4 \mathrm{PBS}$.

A

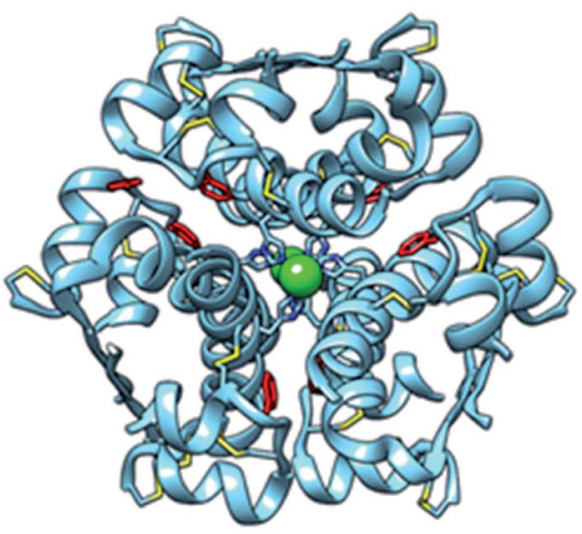

B

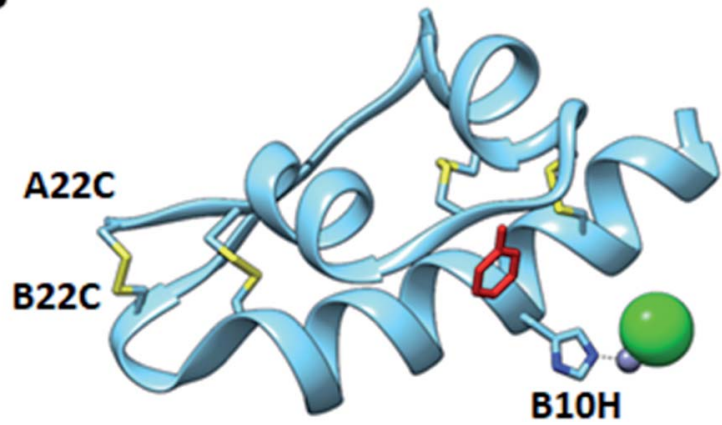

Fig. 3 Crystal structure of A22-B22-4SS-Ins. (A) $R_{6}$ hexamer; (B) one of the A22-B22-4SS-Ins subunits from the hexamer. Positions of phenol ligands (red), Zn (gray) and $\mathrm{Cl}$ ions (green), coordinating histidine residues, and disulfide bonds (yellow) are shown.

square deviation (RMSD) between equivalent $\mathrm{C}_{\alpha}$ atoms of our structure and two wild-type human insulin $\mathrm{R}_{6}$ hexamers, PDB entries $1 \mathrm{ZNJ}$ and $1 \mathrm{EVR},{ }^{14}$ is only $0.40 \AA$ and $0.51 \AA$ across 21 residues of the A chain, and $0.39 \AA$ and $0.36 \AA$ across 29 residues of the B chain, respectively.

An important structural element of insulin is the type-I $\beta$ turn formed by residues B20-B22. Biochemical and structural experiments have shown that this $\beta$-turn is critical for the stability and activity of insulin. ${ }^{19}$ For instance, a modification that ablates this turn through the incorporation of $(\alpha, \beta)$ - dehydrophenylalanine results in an aggregation-prone analog, while the converse modification of rigidifying the turn by substituting the B20 and B22 glycines with D-alanine to reinforce the positive $\varphi$-angles at those positions diminishes activity. These results are consistent with structures of insulin in complex with its receptor, which reveal an induced-fit binding mechanism in which the C-terminal $\beta$-strand of the insulin B-chain hinges away from the hydrophobic core of the hormone. ${ }^{19-21}$ These observations underscore the refractory nature of insulin to mutations in this region of the B-chain. In agreement with the native-like activity of A22-B22-4SS-Ins, alignment of our structure with wild-type insulin shows that residues B20-B23 closely match the corresponding backbone conformation of wildtype insulin despite the incorporation of a new disulfide connecting the C-terminus of the A-chain to the loop of the $\beta$-turn (Fig. 5 and S1†). Further, and consistent with wildtype insulin structures, residues comprising the $\beta$-turn have

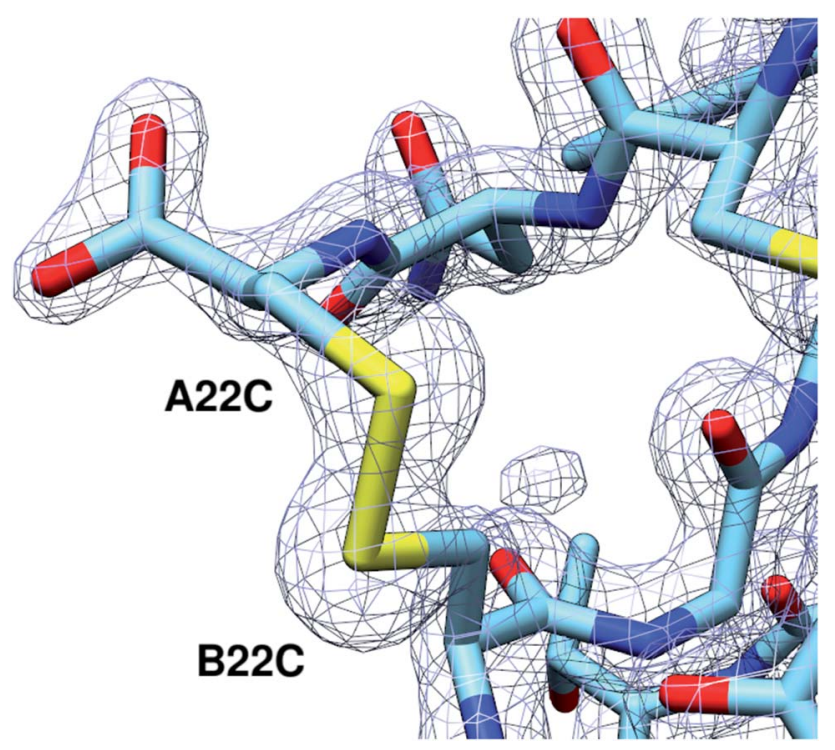

Fig. 4 The A22-B22 disulfide bond is clearly defined. 2Fo-Fc electron density map of the A22-B22 disulfide bond at a contour level of $1.8 \AA$ RMSD. 


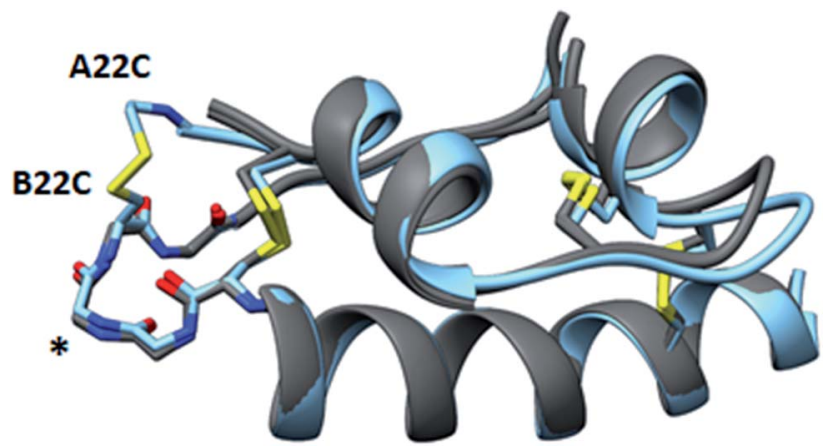

Fig. 5 Structural similarity of A22-B22-4SS-Ins and native insulins. Overlay of A22-B22-4SS-Ins (cyan) with wild-type structures (PDB $1 E V R$ and $1 Z N J$; both gray) with the backbone type-1 beta-turn depicted on the left side of the structure (asterisk).

locally elevated thermal B-factors, indicating that the flexibility of the $\beta$-turn is not hindered by the A22-B22 disulfide bond.

Because residues near the C-terminus of the $\mathrm{B}$ chain are important for insulin dimerization and for binding to the insulin receptors, we characterized each of these properties for A22-B22-4SS-Ins. We used isothermal calorimetry to measure the dimer forming tendency, and found that A22-B22-4SS-Ins has a similar dimer formation tendency as native insulin (Fig. S2 $\dagger$ ). As noted above, A22-B22-4SS-Ins and native insulin give equivalent phosphorylation of Atk (Fig. 2A). We also determined signaling through each of the IR-A and IR-B insulin receptor isoforms by quantification of receptor autophosphoryation. As for native insulin, ${ }^{22}$ signaling was essentially identical for the two isoforms (Fig. S3 $\dagger$ ). In summary, we have demonstrated that the C-terminal of B chain in A22-B22-4SS-Ins is structurally and functionally similar to that of native insulin.

\section{Comparison of A22-B22-4SS-Ins with A10-B4-4SS-Ins}

In addition to the A22-B22-4SS-Ins characterized by this work, one other fully-active, four disulfide human insulin has been reported in the literature, with cysteine mutations connecting A10 and B4. ${ }^{8}$ This analog (A10-B4-4SS-Ins) has improved stability relative to native insulin but displayed altered structural characteristics. The crystal structure of A10-B4-4SS-Ins displays a register shift that results in the formation of a new $\beta$-sheet between B2-B4 and A10-A12 in one monomer in the insulin dimer. Additionally, CD spectroscopy indicated resistance of A10-B4-4SS-Ins to adoption of the phenol-induced Rstate conformation. In contrast, the structure of A22-B22-4SSIns shows fewer structural alterations resulting from the new disulfide bond, a property that could be favorable for the development of novel, ultra-stable therapeutic insulins.

\section{Conclusion}

Our results demonstrate that tethering between the C-terminal A chain and the B21-B23 region can lead to bioactivity variation ranging from retained potency to 50 -fold reduction. Further, we report A22-B22-4SS-Ins as a novel insulin analog with retained potency, increased aggregation stability, and a crystal structure that is closely similar to native insulin. Our findings thereby complement recent efforts in generating four-disulfide insulin $^{9,10}$ and single-chain insulin, ${ }^{23,24}$ and advance efforts to develop a much more stable therapeutic insulin. Further investigations regarding insulin signaling pathway activation in vitro and in vivo are underway.

\section{Conflicts of interest}

There are no conflicts to declare.

\section{Acknowledgements}

We thank NIDDK (R01DK120430) and JDRF (5-CDA-2018-572 AN) for funding support. X. X. is a JDRF postdoctoral fellow. Use of the Stanford Synchrotron Radiation Lightsource, SLAC National Accelerator Laboratory, is supported by the U.S. Department of Energy, Office of Science, Office of Basic Energy Sciences under Contract No. DE-AC02-76SF00515. The SSRL Structural Molecular Biology Program is supported by the DOE Office of Biological and Environmental Research, and by the National Institutes of Health, National Institute of General Medical Sciences. UCSF Chimera, which was used for molecular representations and analyses, was developed by the Resource for Biocomputing, Visualization, and Informatics at the University of California, San Francisco, with support from NIH P41-GM103311.

\section{References}

1 A. N. Zaykov, J. P. Mayer and R. D. DiMarchi, Pursuit of a perfect insulin, Nat. Rev. Drug Discovery, 2016, 15(6), 425439.

2 M. A. Weiss, The structure and function of insulin: decoding the TR transition, Vitam. Horm., 2009, 80, 33-49.

3 Q. X. Hua, S. H. Nakagawa, W. Jia, K. Huang, N. B. Phillips, S. Q. Hu and M. A. Weiss, Design of an active ultrastable single-chain insulin analog: synthesis, structure, and therapeutic implications, J. Biol. Chem., 2008, 283(21), 14703-14716.

$4 \mathrm{~J}$. M. Conlon, Evolution of the insulin molecule: insights into structure-activity and phylogenetic relationships, Peptides, 2001, 22(7), 1183-1193.

5 H. Safavi-Hemami, J. Gajewiak, S. Karanth, S. D. Robinson, B. Ueberheide, A. D. Douglass, A. Schlegel, J. S. Imperial, M. Watkins, P. K. Bandyopadhyay, M. Yandell, Q. Li, A. W. Purcell, R. S. Norton, L. Ellgaard and B. M. Olivera, Specialized insulin is used for chemical warfare by fishhunting cone snails, Proc. Natl. Acad. Sci. U. S. A., 2015, 112(6), 1743-1748.

6 F. Wu, J. P. Mayer, V. M. Gelfanov, F. Liu and R. D. DiMarchi, Synthesis of Four-Disulfide Insulin Analogs via Sequential Disulfide Bond Formation, J. Org. Chem., 2017, 82(7), 35063512.

7 J. Mansfeld, G. Vriend, B. W. Dijkstra, O. R. Veltman, B. Van den Burg, G. Venema, R. Ulbrich-Hofmann and V. G. Eijsink, 
Extreme stabilization of a thermolysin-like protease by an engineered disulfide bond, J. Biol. Chem., 1997, 272(17), 11152-11156.

8 T. N. Vinther, M. Norrman, U. Ribel, K. Huus, M. Schlein, D. B. Steensgaard, T. A. Pedersen, I. Pettersson, S. Ludvigsen, T. Kjeldsen, K. J. Jensen and F. Hubalek, Insulin analog with additional disulfide bond has increased stability and preserved activity, Protein Sci., 2013, 22(3), 296-305.

9 T. N. Vinther, I. Pettersson, K. Huus, M. Schlein, D. B. Steensgaard, A. Sorensen, K. J. Jensen, T. Kjeldsen and F. Hubalek, Additional disulfide bonds in insulin: Prediction, recombinant expression, receptor binding affinity, and stability, Protein Sci., 2015, 24(5), 779-788.

10 F. M. Brunel, J. P. Mayer, V. M. Gelfanov, A. N. Zaykov, B. Finan, D. Perez-Tilve and R. D. DiMarchi, A disulfidescan of insulin by $[3+1]$ methodology exhibits site-specific influence on bioactivity, ACS Chem. Biol., 2019, 14(8), 1829-1835.

11 P. Ahorukomeye, M. M. Disotuar, J. Gajewiak, S. Karanth, M. Watkins, S. D. Robinson, P. Florez Salcedo, N. A. Smith, B. J. Smith, A. Schlegel, B. E. Forbes, B. Olivera, D. HungChieh Chou and H. Safavi-Hemami, Fish-hunting cone snail venoms are a rich source of minimized ligands of the vertebrate insulin receptor, Elife, 2019, 8, e41574.

12 A. M. Brzozowski, E. J. Dodson, G. G. Dodson, G. N. Murshudov, C. Verma, J. P. Turkenburg, F. M. de Bree and Z. Dauter, Structural origins of the functional divergence of human insulin-like growth factor-I and insulin, Biochemistry, 2002, 41(30), 9389-9397.

13 D. T. Dransfield, E. H. Cohen, Q. Chang, L. G. Sparrow, J. D. Bentley, O. Dolezal, X. Xiao, T. S. Peat, J. Newman, P. A. Pilling, T. Phan, I. Priebe, G. V. Brierley, N. Kastrapeli, K. Kopacz, D. Martik, D. Wassaf, D. Rank, G. Conley, Y. Huang, T. E. Adams and L. Cosgrove, A human monoclonal antibody against insulin-like growth factor-II blocks the growth of human hepatocellular carcinoma cell lines in vitro and in vivo, Mol. Cancer Ther., 2010, 9(6), 1809-1819.

14 G. D. Smith, E. Ciszak, L. A. Magrum, W. A. Pangborn and R. H. Blessing, $\mathrm{R}_{6}$ hexameric insulin complexed with $\mathrm{m}-$ cresol or resorcinol, Acta Crystallogr., Sect. D: Biol. Crystallogr., 2000, 56(12), 1541-1548.

15 F. Liu, E. Y. Luo, D. B. Flora and A. R. Mezo, A synthetic route to human insulin using isoacyl peptides, Angew. Chem., Int. Ed. Engl., 2014, 53(15), 3983-3987.
16 M. J. Webber, E. A. Appel, B. Vinciguerra, A. B. Cortinas, L. S. Thapa, S. Jhunjhunwala, L. Isaacs, R. Langer and D. G. Anderson, Supramolecular PEGylation of biopharmaceuticals, Proc. Natl. Acad. Sci. U. S. A., 2016, 113(50), 14189-14194.

17 S. Rahuel-Clermont, C. A. French, N. C. Kaarsholm, M. F. Dunn and C. I. Chou, Mechanisms of stabilization of the insulin hexamer through allosteric ligand interactions, Biochemistry, 1997, 36(19), 5837-5845.

$18 \mathrm{H}$. Berchtold and R. Hilgenfeld, Binding of phenol to R6 insulin hexamers, Biopolymers, 1999, 51(2), 165-172.

19 J. G. Menting, Y. Yang, S. J. Chan, N. B. Phillips, B. J. Smith, J. Whittaker, N. P. Wickramasinghe, L. J. Whittaker, V. Pandyarajan, Z. L. Wan, S. P. Yadav, J. M. Carroll, N. Strokes, C. T. Roberts Jr, F. Ismail-Beigi, W. Milewski, D. F. Steiner, V. S. Chauhan, C. W. Ward, M. A. Weiss and M. C. Lawrence, Protective hinge in insulin opens to enable its receptor engagement, Proc. Natl. Acad. Sci. U. S. A., 2014, 111(33), E3395-E3404.

20 G. Scapin, V. P. Dandey, Z. Zhang, W. Prosise, A. Hruza, T. Kelly, T. Mayhood, C. Strickland, C. S. Potter and B. Carragher, Structure of the insulin receptor-insulin complex by single-particle cryo-EM analysis, Nature, 2018, 556(7699), 122-125.

21 F. Weis, J. G. Menting, M. B. Margetts, S. J. Chan, Y. Xu, N. Tennagels, P. Wohlfart, T. Langer, C. W. Muller, M. K. Dreyer and M. C. Lawrence, The signalling conformation of the insulin receptor ectodomain, Nat. Commun., 2018, 9(1), 4420.

22 R. Slaaby, Specific insulin/IGF1 hybrid receptor activation assay reveals IGF1 as a more potent ligand than insulin, Sci. Rep., 2015, 5, 7911.

23 M. D. Glidden, K. Aldabbagh, N. B. Phillips, K. Carr, Y. S. Chen, J. Whittaker, M. Phillips, N. P. Wickramasinghe, N. Rege, M. Swain, Y. Peng, Y. Yang, M. C. Lawrence, V. C. Yee, F. Ismail-Beigi and M. A. Weiss, An ultra-stable single-chain insulin analog resists thermal inactivation and exhibits biological signaling duration equivalent to the native protein, J. Biol. Chem., 2018, 293(1), 47-68.

24 M. Y. Jeong, J. Rutter and D. H. Chou, Display of SingleChain Insulin-like Peptides on a Yeast Surface, Biochemistry, 2019, 58(3), 182-188. 